\title{
Vinflunine for the treatment of advanced or metastatic transitional cell carcinoma of the urothelial tract: an evidence-based review of safety, efficacy, and place in therapy
}

This article was published in the following Dove Press journal: Core Evidence

\author{
Steven C Brousell' \\ Joseph J Fantony' \\ Megan G Van Noord ${ }^{2}$ \\ Michael R Harrison ${ }^{3}$ \\ Brant A Inman' \\ 'Division of Urology, ${ }^{2}$ Division of \\ Research and Education Services, \\ ${ }^{3}$ Division of Medical Oncology, Duke \\ University Medical Center, Durham, \\ NC, USA
}

Correspondence: Brant A Inman Division of Urology, Duke University Medical Center, Box 103868, 3007 Snyderman Building, 905 La Salle Street, Durham, NC 277I0, USA

$\mathrm{Tel}+\mathrm{I} 9196841322$

Fax +19196687093

Email brant.inman@duke.edu
Background: A systematic review and meta-analysis of the use of systemic vinflunine (VIN) in the treatment of urothelial carcinoma (UC) was performed to evaluate its efficacy based on current available clinical data.

Methods: This review was prospectively registered at the International Prospective Register of Systematic Reviews, PROSPERO (registration CRD42016049294). Electronic databases including MEDLINE ${ }^{\circledR}$, Embase $^{\circledR}$, Cochrane Central Register of Controlled Trials, and Web of Science were searched through December 2016. We performed a meta-analysis of the published data. Primary end points were progression-free survival (PFS) and overall survival (OS). Numerous secondary clinical outcomes were analyzed including response and toxicity data.

Results: We identified 382 publications, of which 35 met inclusion criteria for this review representing 29 unique studies. A total of 2,255 patients received VIN for the treatment of UC in the included studies. OS and PFS were analyzed in a pooled Kaplan-Meier analysis. Response data were available for 1,416 VIN-treated patients with random effects proportion of complete response in $1 \%$, partial response in $18 \%$, and overall response rate of $21 \%$. Toxicity analysis revealed fatigue $(40.1 \%)$, nausea $(33.9 \%)$, constipation $(34.1 \%)$, and alopecia $(26.0 \%)$ as the most prevalent overall non-hematologic adverse events (AEs). Most prevalent grade 3-4 AEs were fatigue (10.2\%), abdominal pain (8.2\%), myalgias $(2.5 \%)$, and nausea $(2.3 \%)$. Most common hematologic AEs of all grades were anemia (56.6\%), neutropenia (46.0\%), thrombocytopenia (25.5\%), and febrile neutropenia (6.6\%). Grade 3-4 hematologic AEs had the following pooled rates: neutropenia, $24.6 \%$; anemia, $10.2 \%$; febrile neutropenia, $5.4 \%$; and thrombocytopenia, $3.0 \%$.

Conclusion: VIN has been explored as a combination first-line treatment as well as a singleagent second-line, third-line, and maintenance therapy for advanced and metastatic UC. In firstline treatment of UC, either as a maintenance agent after cisplatin or as a primary combination therapy, VIN may be a promising alternative to current treatments. Further studies are needed to compare first-line combination VIN regimens to the current standard of care in order to assess long-term survival outcomes. Second- and third-line VIN monotherapy does provide a proven, although limited, survival benefit in platinum-refractory patients.

Keywords: vinflunine, urothelial carcinoma, bladder cancer, metastatic, survival, chemotherapy 


\section{Core evidence clinical impact summary for vinflunine in advanced and metastatic urothelial carcinoma}

\begin{tabular}{|c|c|c|}
\hline $\begin{array}{l}\text { Outcome } \\
\text { measure }\end{array}$ & Evidence & Implication \\
\hline $\begin{array}{l}\text { Disease- } \\
\text { oriented } \\
\text { evidence }\end{array}$ & $\begin{array}{l}\text { Clinical trials and } \\
\text { retrospective analyses }\end{array}$ & $\begin{array}{l}\text { Vinflunine when administered as second-line treatment } \\
\text { for stage IV UC demonstrated modest gains in tumor } \\
\text { response rate and survival }\end{array}$ \\
\hline $\begin{array}{l}\text { Patient- } \\
\text { oriented } \\
\text { evidence }\end{array}$ & $\begin{array}{l}\text { Clinical trials and } \\
\text { retrospective analyses }\end{array}$ & $\begin{array}{l}\text { Risk of severe AEs must be weighed carefully against } \\
\text { the modest survival benefit of VIN in advanced UC. } \\
\text { However, most AEs are preventable or treatable }\end{array}$ \\
\hline $\begin{array}{l}\text { Economic } \\
\text { evidence }\end{array}$ & $\begin{array}{l}\text { Multicenter, observational, } \\
\text { retrospective cohort study }\end{array}$ & $\begin{array}{l}\text { VIN median treatment costs are } € 13,096 \text { per patient, } \\
€ 44,789 \text { per progression-free year gained, and } € 22,750 \\
\text { per life-year granted }\end{array}$ \\
\hline
\end{tabular}

\section{Introduction}

Metastatic urothelial carcinoma (UC) is an aggressive and highly lethal cancer despite many patients having an initial response to current systemic cytotoxic therapies. Current first-line treatment for advanced UC consists of platinumbased combination chemotherapy regimens. Of these, cisplatin-based combinations have demonstrated the most favorable overall survival (OS) - 12-15 months approximately - and are widely considered the standard of care. ${ }^{1}$ Patients who are cisplatin ineligible, most often due to renal insufficiency or neurological disease, traditionally received carboplatin-based combination regimens which, although more tolerable, appear to have worse survival outcomes and are therefore considered less effective. ${ }^{2}$ Most patients receiving platinum regimens for metastatic UC will ultimately progress, and many will be offered second-line systemic therapy. Recently, PD-L1 and PD-1 immune checkpoint inhibitors have been approved by the US Food and Drug Administration (FDA) for second-line treatment of advanced $\mathrm{UC}$ and for patients in first line who are cisplatin ineligible.

In 2009, the European Medical Association approved vinflunine (VIN), the newest member of the vinca alkaloid family, as a second-line therapy for advanced UC after platinum failure. This drug prevents microtubule assembly during mitosis, inducing apoptosis. ${ }^{3,4}$ Early European Phase II trials in platinum-refractory metastatic UC examined the activity of VIN in 51 and 175 patients showing a response rate of $18 \%$ and $15 \%$, respectively, with median progression-free survival (PFS) and OS of 3.0 and 6.6 months in the first study and 2.8 and 8.2 months in the second study. ${ }^{5,6}$ These results led to a pivotal, multicenter Phase III randomized controlled trial by Bellmunt et al comparing VIN plus best supportive care (BSC) to $\mathrm{BSC}$ alone in platinum-refractory advanced UC. ${ }^{7,8}$ This trial of 370 patients receiving second-line VIN showed a modest survival benefit of 2.3 months above BSC alone. The FDA has not approved VIN for treatment of advanced UC at this time.
JASiMA, a small 20-patient international Phase II trial, examined response data in patients with advanced UC receiving maintenance VIN immediately following gemcitabinecisplatin first-line therapy and showed enhanced response in 5 patients (27.8\%). ${ }^{9}$ MAJA, a placebo-controlled Phase II trial, examined maintenance VIN versus BSC in a larger cohort (88) of patients with platinum-responsive advanced UC and found improved PFS of 6.5 months in the VIN arm versus 4.6 months in BSC arm (HR 0.56, 95\% CI 0.34-0.93, $p=0.024) .{ }^{10}$

The JASINT1 international Phase II trial examined first-line VIN combination therapy with either vinfluninegemcitabine (VG) or vinflunine-carboplatin (VC) in 69 patients with advanced UC who were ineligible to receive cisplatin. This study found similar disease control rates (DCRs), overall response rates (ORRs), and OS between the 2 groups. ${ }^{11}$ Lastly, the IMvigor 211 study has been presented in abstract form and included 250 patients treated with VIN as part of a cohort patients with platinum-refractory metastatic UC receiving chemotherapy versus atezolizumab. ${ }^{12}$

The objective of this study was to systematically amass all relevant publications to evaluate the efficacy of VIN in adult patients with UC in terms of response and survival rates as well as toxicity and tolerability of this therapy.

\section{Methods \\ Protocol registration}

Prior to the formal literature search, the protocol was prospectively registered at the International Prospective Register of Systematic Reviews, PROSPERO (registration CRD42016049294).

\section{Study selection criteria}

To be eligible, retrieved articles had to include subjects (1) aged $\geq 18$ years, (2) with a diagnosis of UC of the bladder, ureter, urethra, or renal pelvis, and (3) who were treated with systemic VIN chemotherapy. Studies that dealt primarily with animals 
or the treatment of non-urothelial malignancies were excluded. All studies, whether retrospective or prospective, whether randomized or not, were initially eligible, with the exception of single-patient case reports which were excluded. To be included in analyses, however, studies had to specifically report efficacy or toxicity outcomes for those subjects receiving VIN.

\section{Search strategy for identification of studies}

The initial literature search was designed and executed by a medical information specialist (MVN). We searched MEDLINE $^{\circledR}$, Embase $^{\circledR}$, Cochrane Central Register of Controlled Trials, and Web of Science electronic databases through December 2016. The search captured terms for VIN and urinary bladder neoplasms using subject headings and text words. The search strategies are included in the Supplementary materials, Appendix 1. Reference lists of included series were manually screened for any additional studies to minimize retrieval bias. All identified manuscripts and their citation information were imported into an electronic database.

\section{Critical evaluation of the selected studies}

All manuscripts retrieved by the search strategy had their title and abstract prescreened by 2 of the researchers. Any reference deemed to potentially meet any of the inclusion criteria was tagged for full-text screening. A third reviewer adjudicated any prescreening discrepancies occurring between the first 2 reviewers. Full-text manuscripts were retrieved (where possible) for all prescreened abstracts, and these manuscripts were reviewed in detail by 2 researchers to determine if they met inclusion/exclusion criteria for the systematic review. In some instances, conference abstracts were identified without any discernible companion manuscript and such abstracts were retained if sufficient detail was present in the abstract for later data abstraction. No studies were excluded based on perceived quality or bias. Manuscripts (and in some instances abstracts) meeting screening criteria were then subject to data abstraction.

\section{Data abstraction}

Data elements were abstracted by 2 independent reviewers from the screened manuscripts and abstracts into an electronic database. Discrepancies in abstracted data were resolved by consensus review of the manuscript. All data were extracted directly from the manuscript or calculated from the available information when necessary. Clinical data including trial details, patient-specific data (age, sex, performance status), disease-specific data (tumor stage, tumor location, location of metastases, prior treatment, associated lab abnormalities), response data (complete response [CR], partial response [PR], stable disease, objective response rate, duration of response), survival outcomes, and toxicity outcomes were collected. The data on all trials were based on the intentionto-treat (ITT) principle whenever possible.

The primary efficacy outcomes analyzed were median PFS and OS, treatment response, and response duration. Response rates were generally reported according to RECIST criteria. ${ }^{13}$ In this system, progressive disease is defined as the development of new lesions or growth of existing lesions $\geq 20 \%$; a CR indicates the complete disappearance of tumor; a PR indicates that the tumor has shrunk by $\geq 30 \%$ but is still visible on imaging; the objective response rate is the sum of the CR and PR rates; and stable disease is when tumor size shrinks by $<30 \%$ and grows by no more than $20 \%$.

Safety outcome analysis included incidence, type, and (where possible) the Common Terminology Criteria for Adverse Events (CTCAE) severity of non-hematologic adverse events (AEs) (fatigue, nausea, vomiting, mucositis, abdominal pain, constipation, myalgia, neuropathy, alopecia, infusion-site reaction) and hematologic AEs (anemia, neutropenia, neutropenic fever, thrombocytopenia). ${ }^{14}$

\section{Analysis and presentation of results}

Abstracted data were cleaned and condensed. Trials that generated multiple publications were combined into a single consensus record per trial. This resulted in 29 total studies that contain data analyzable in our systematic review. When individual publications from the same trial had different outcome data, we selected the outcomes with 1) the longest median follow-up time and 2) the data from the publication describing the full set of subjects and toxicity outcomes (i.e., not subgroup publications) for inclusion in the consensus record.

Statistical analysis was done using R 3.4.2 "Short Summer" on RStudio 1.1.383. For pooling proportions, the meta package was used. ${ }^{15}$ Fixed effect (FE) and random effect (RE) models were constructed using inverse variance weighting. For variance stabilization, proportions were pooled after arcsine transformation, ${ }^{16}$ restricted maximum likelihood estimation was used to calculate the between-study variance $\left(\tau^{2}\right)$ in the RE models, and pooled arcsine-transformed proportions and their 95\% CIs were then back-transformed to the normal scale for presentation. ${ }^{17} \mathrm{CIs}$ for individual study proportions were calculated using the Clopper-Pearson method. The presence of residual heterogeneity was assessed using the $I^{2}$ statistic. ${ }^{18}$ Forest plots were created to visually demonstrate results. To measure and plot survival times across studies, individual Kaplan-Meier plots were extracted and digitized using the digitize package for $\mathrm{R}$ and plotted using ggplot $2 .{ }^{19,20}$ 


\section{Results}

\section{Search results}

Figure 1 presents the flow of identification and inclusion of articles as recommended by the Preferred Reporting Items for Systematic Reviews and Meta-Analysis statement. ${ }^{21}$ Our initial search resulted in 381 publications after deduplication. After title and abstract review, 198 publications were excluded, leaving 84 studies for full-text analysis. Upon full-text analysis, 50 additional publications were excluded for various reasons ( 28 were duplicate data sets or articles, 16 lacked VIN data, 3 did not involve UC, 2 were not available in English, and 1 was a review article). One trial, IMvigor211, was presented in abstract form after our planned inclusion date range, but was included because it was felt to be an important study and contained a large number of VIN-treated patients. This resulted in 35 publications from which data variables were abstracted, and characteristics of these studies are shown in Table 1. The final analysis included 29 single consensus records for which data were available.

\section{Chemotherapy with VIN}

A total of 2,255 subjects received systemic therapy with VIN for the treatment of UC. All patients had the American Join Committee on Cancer stage IV cancer, though not all were metastatic as some were locally advanced nonmetastatic patients. VIN standard dose was most commonly $320 \mathrm{mg} / \mathrm{m}^{2}$ every 21 days as a 20-minute infusion (reported in 17 of 28 included studies) with reduced doses of either 250 or 280 $\mathrm{mg} / \mathrm{m}^{2}$ with similar administration schedule. The majority of VIN administration was as second-line therapy after platinum failure; however, studies included data on third-line, maintenance, and first-line combination therapy. ${ }^{9-11,22-25}$

\section{Response data}

VIN chemotherapy response information was available from 17 studies, representing 1,416 patients. Forest plots for CR (Figure 2), PR (Figure 3), and overall response (Figure 4) are presented. There were few documented complete responders, and the pooled CR rate using FEs was 1\% (95\% CI 1-2\%) and

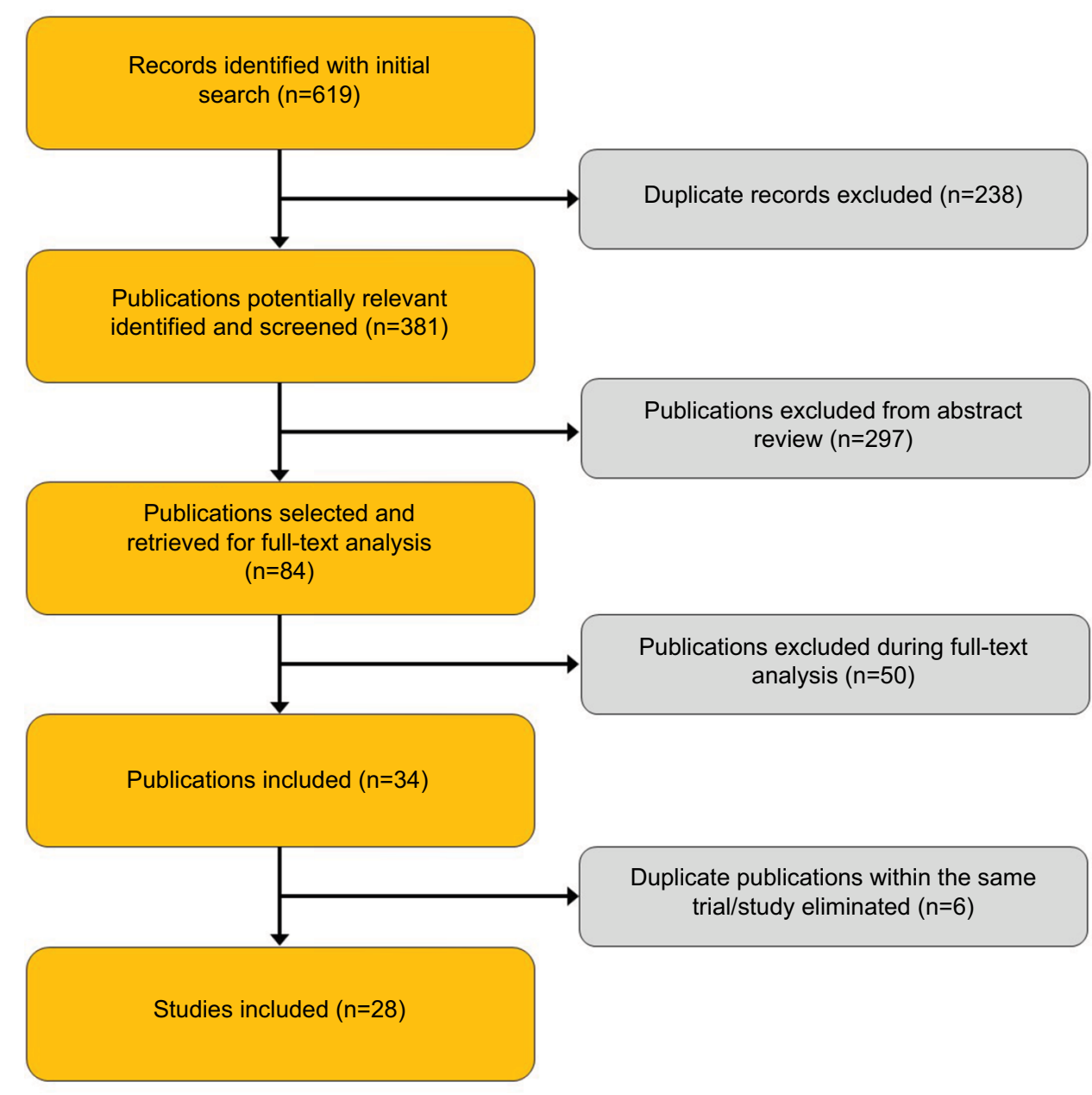

Figure I Study selection flow chart. 


\begin{tabular}{|c|c|}
\hline Source & Proportion (95\% \\
\hline Bellmunt et $\mathrm{al}^{8}$ & $0.00[0.00-0.01]$ \\
\hline Castellano et al ${ }^{35}$ & $0.02[0.00-0.07]$ \\
\hline Chirivella et $\mathrm{al}^{39}$ & $0.02[0.00-0.12]$ \\
\hline Culine et $\mathrm{al}^{5}$ & $0.00[0.00-0.07]$ \\
\hline De Santis et al ${ }^{11}$ & $0.12[0.05-0.22]$ \\
\hline Di Lorenzo et al ${ }^{23}$ & $0.00[0.00-0.31]$ \\
\hline Facchini et $\mathrm{al}^{27}$ & $0.00[0.00-0.08]$ \\
\hline Hegele et al ${ }^{44}$ & $0.05[0.00-0.24]$ \\
\hline Holmsten et al ${ }^{45}$ & $0.01[0.00-0.05]$ \\
\hline Hussain et al ${ }^{47}$ & $0.00[0.00-0.09]$ \\
\hline Medioni et al ${ }^{36}$ & $0.05[0.02-0.10]$ \\
\hline Moriceau et al ${ }^{48}$ & $0.00[0.00-0.18]$ \\
\hline Palacka et $\mathrm{al}^{26}$ & $0.00[0.00-0.21]$ \\
\hline Passalacqua et al ${ }^{49}$ & $0.03[0.01-0.07]$ \\
\hline Pistamaltzian et al ${ }^{50}$ & $0.03[0.00-0.10]$ \\
\hline Retz et al ${ }^{51}$ & $0.05[0.01-0.13]$ \\
\hline Vaughn et al ${ }^{6}$ & $0.00[0.00-0.02]$ \\
\hline Total (fixed effect) & $0.01[0.01-0.02]$ \\
\hline Total (random effects) & $0.01[0.00-0.03]$ \\
\hline Heterogeneity: $\chi_{16}^{2}=62$. & $(P<0.01), l^{2}=75 \%$ \\
\hline
\end{tabular}

Figure 2 Forest plot of complete response data.

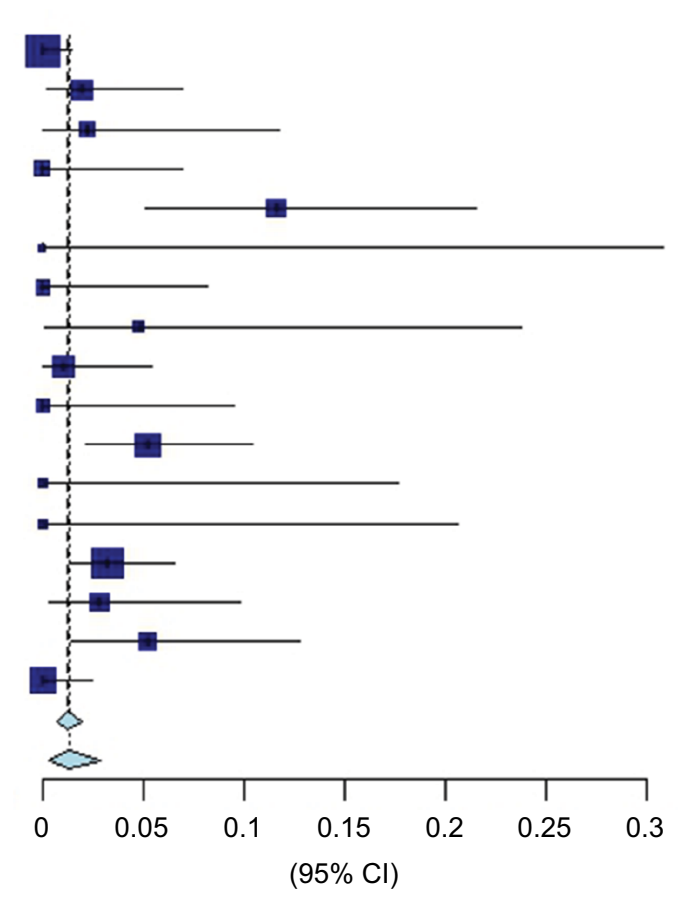

$(95 \% \mathrm{Cl})$

\begin{tabular}{|c|c|}
\hline Source & Proportion $(95 \% \mathrm{Cl})$ \\
\hline Bellmunt et al ${ }^{8}$ & $0.09[0.06-0.13]$ \\
\hline Castellano et al 35 & $0.23[0.15-0.32]$ \\
\hline Chirivella et al ${ }^{39}$ & $0.24[0.13-0.40]$ \\
\hline Culine et $\mathrm{al}^{5}$ & $0.18[0.08-0.31]$ \\
\hline De Santis et al ${ }^{11}$ & $0.36[0.25-0.49]$ \\
\hline Di Lorenzo et al ${ }^{23}$ & $0.30[0.07-0.65]$ \\
\hline Facchini et al ${ }^{27}$ & $0.12[0.04-0.25]$ \\
\hline Hegele et al ${ }^{44}$ & $0.14[0.03-0.36]$ \\
\hline Holmsten et al ${ }^{45}$ & $0.22[0.14-0.31]$ \\
\hline Hussain et al ${ }^{47}$ & 0.35 [0.20-0.53] \\
\hline Medioni et al ${ }^{36}$ & $0.17[0.11-0.25]$ \\
\hline Moriceau et al48 & $0.32[0.13-0.57]$ \\
\hline Palacka et al ${ }^{26}$ & $0.12[0.02-0.38]$ \\
\hline Passalacqua et al 49 & $0.10[0.06-0.15]$ \\
\hline Pistamaltzian et al50 & $0.10[0.06-0.15]$ \\
\hline Retz et al ${ }^{51}$ & $0.18[0.10-0.29]$ \\
\hline Vaughn et $\mathrm{al}^{6}$ & $0.15[0.09-0.21]$ \\
\hline Total (fixed effect) & $0.16[0.14-0.18]$ \\
\hline Total (random effects) & $0.18[0.14-0.22]$ \\
\hline Heterogeneity: $\chi_{16}^{2}=50$ & $30(P<0.01), P^{2}=72 \%$ \\
\hline
\end{tabular}

Figure 3 Forest plot of partial response data.

using REs was $1 \%$ (95\% CI $1-3 \%$ ), with an $I^{2}$ of $75 \%$. The pooled PR rate was $16 \%$ (95\% CI 14-18\%) using FEs and $18 \%$ (95\% CI 14-22\%) using REs, with an $I^{2}$ of $72 \%$. The pooled objective response rate with FEs was 18\% (95\% CI 16-20\%) and with REs was $21 \%$ (95\% CI 16-26\%) with an $I^{2}$ of $79 \%$.

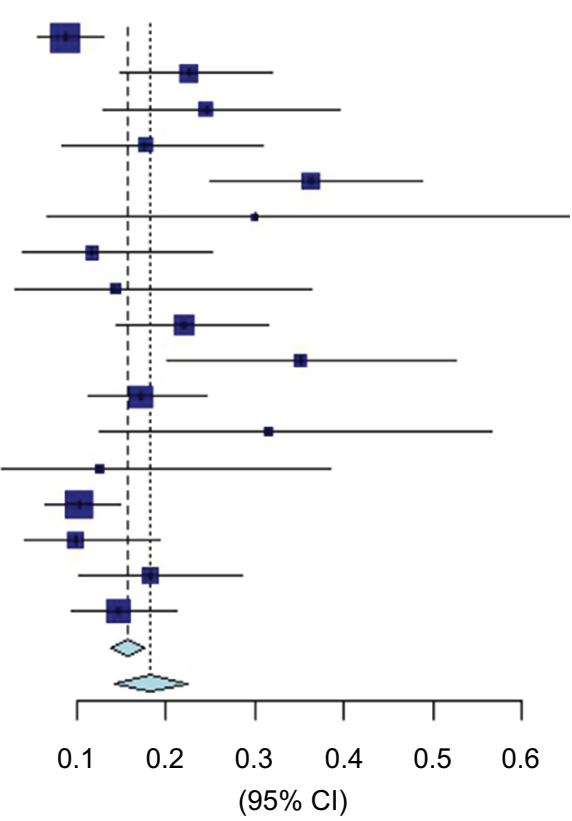

\section{Survival analysis}

Kaplan-Meier curves were available for OS for 11 studies (Figure 5), and median OS ranged from 5.2 to 13.4 months. ${ }^{11,26}$ PFS was available for 6 studies (Figure 6) and ranged from 2.2 to 6.5 months..$^{10,27}$ 
Table I Study-level characteristics

\begin{tabular}{|c|c|c|c|c|c|c|}
\hline Author & Country of Origin & $\begin{array}{l}\text { Year of } \\
\text { Publication }\end{array}$ & Journal & Centers & Blinding & Study Type \\
\hline Aparicio et $\mathrm{al}^{|3|}$ & Spain & 2013 & European Journal of Cancer & multicenter & open label & case series \\
\hline Bamias et $\mathrm{al}^{38}$ & Greece & 2016 & Clinical Genitourinary Cancer & multicenter & open label & case series \\
\hline Bellmunt et $\mathrm{al}^{28}$ & Spain & 2010 & J Clin Oncol & multicenter & open label & $\mathrm{RCT}$ \\
\hline Bellmunt et $\mathrm{al}^{7}$ & Spain & 2013 & Ann Oncol & multicenter & open label & RCT \\
\hline Bellmunt et $\mathrm{a}^{8}$ & Spain & 2009 & J Clin Oncol & multicenter & open label & $\mathrm{RCT}$ \\
\hline Bellmunt et $\mathrm{al}^{22}$ & Spain & 2015 & Journal & multicenter & open label & $\mathrm{RCT}$ \\
\hline Castellano et a ${ }^{35}$ & Spain & 2014 & BMC Cancer & multicenter & open label & case series \\
\hline Chirivella et $\mathrm{a}^{39}$ & Spain & 2013 & European Journal of Cancer & multicenter & open label & case series \\
\hline Culine et $\mathrm{al}^{5}$ & UK & 2006 & $\mathrm{Br} J$ Cancer & multicenter & open label & phase II trial \\
\hline De Santis et al" & Austria & 2016 & Ann Oncol & multicenter & open label & phase II trial \\
\hline De Wit et al ${ }^{9}$ & Germany & 2015 & European Journal of Cancer & multicenter & open label & phase II trial \\
\hline Di Lorenzo et $\mathrm{a}^{23}$ & Italy & 2015 & Medicine (Baltimore) & multicenter & open label & case series \\
\hline Di Palma et $a^{29}$ & France & 2013 & European Journal of Cancer & multicenter & open label & case series \\
\hline Donini et $\mathrm{al}^{40}$ & Italy & 2015 & Annals of Oncology & multicenter & open label & case series \\
\hline Facchini et $\mathrm{a}^{27}$ & Italy & 2016 & Front Pharmacol & single center & open label & case series \\
\hline Font et $\mathrm{al}^{10}$ & Spain & 2016 & Journal of Clinical Oncology & multicenter & open label & $\mathrm{RCT}$ \\
\hline George et $\mathrm{al}^{41}$ & USA & 2007 & Ejc Supplements & multicenter & open label & phase II trial \\
\hline Gerullis et $\mathrm{al}^{142}$ & Germany & 2013 & Anticancer Drugs & single center & open label & phase I trial \\
\hline Guglieri-Lopez et a ${ }^{32}$ & Spain & 2015 & Anticancer Drugs & multicenter & open label & Cohort \\
\hline Harshman et a $\left.\right|^{30}$ & USA & 2013 & $\mathrm{Br}$ J Cancer & multicenter & open label & $\mathrm{RCT}$ \\
\hline Hegele et $\mathrm{a}^{43}$ & Germany & 2015 & Journal of Clinical Oncology & multicenter & open label & case series \\
\hline Hegele et $\mathrm{al}^{44}$ & Germany & 2014 & Urol Int & multicenter & open label & case series \\
\hline Holmsten et $\mathrm{al}^{45}$ & Sweden & 2016 & Oncol Lett & multicenter & open label & case series \\
\hline Houede et $\mathrm{a}^{46}$ & France & 2016 & BMC Cancer & multicenter & open label & case series \\
\hline Hussain et $\mathrm{al}^{47}$ & UK & 2015 & European Journal of Cancer & multicenter & open label & case series \\
\hline Marongiu et $\mathrm{al}^{24}$ & Italy & 2013 & European Urology, Supplements & multicenter & open label & case series \\
\hline Medioni et $\mathrm{al}^{36}$ & Italy & 2016 & BMC Cancer & multicenter & open label & case series \\
\hline Moriceau et $\mathrm{al}^{48}$ & France & 2015 & Clin Genitourin Cancer & single center & open label & case series \\
\hline Palacka et al ${ }^{26}$ & Slovak Republic & 2014 & Klin Onkol & single center & open label & case series \\
\hline Passalacqua et $\mathrm{al}^{49}$ & Italy & 2016 & Journal of Clinical Oncology & multicenter & open label & case series \\
\hline Pistamaltzian et $\mathrm{al}^{50}$ & Greece & 2016 & Anticancer Drugs & multicenter & open label & case series \\
\hline Polo et $\mathrm{a}^{25}$ & Spain & 2014 & Journal of Clinical Oncology & multicenter & open label & $\mathrm{RCT}$ \\
\hline Powles et al ${ }^{12}$ & UK & 2017 & EAS Meeting & multicenter & open label & $\mathrm{RCT}$ \\
\hline Retz et $\mathrm{al}^{51}$ & Germany & 2015 & BMC Cancer & multicenter & open label & case series \\
\hline Vaughn et al ${ }^{6}$ & USA & 2009 & Cancer & multicenter & open label & phase II trial \\
\hline
\end{tabular}

Abbreviations: $\mathrm{RCT}$, randomized controlled trial; VIN, vinflunine.

\section{Toxicity analysis}

AEs (overall and grade 3-4) associated with VIN treatment are summarized in Table 2 for non-hematologic events and Table 3 for hematological events. Forest plots for these are found in the Supplementary materials, Appendix 2. The most prevalent non-hematologic AEs according to pooled REs analysis including all CTCAE grades were fatigue (40.1\%), nausea (33.9\%), constipation $(34.1 \%)$, and alopecia $(26.0 \%)$. The most prevalent CTCAE grade 3-4 non-hematologic AEs were fatigue (10.2\%), abdominal pain (8.2\%), myalgias $(2.5 \%)$, and nausea $(2.3 \%)$. The most common hematological AEs were anemia (56.6\%), neutropenia $(46.0 \%)$, thrombocytopenia (25.5\%), and febrile neutropenia (6.6\%). Grade 3-4 hematologic AE rates were $24.6 \%$ for neutropenia, $10.2 \%$ for anemia, $5.4 \%$ for febrile neutropenia, and $3.0 \%$ for thrombocytopenia.

\section{Discussion}

VIN has been evaluated in multiple trials as a first-line, secondline, third-line, and maintenance chemotherapeutic agent in the treatment of advanced and metastatic UC. The largest multinational study to date looking at VIN plus BSC versus BSC alone showed that VIN treatment as a second-line agent in platinum-refractory patients provides a 2.3-month improvement in median OS. With $>40$ months of follow-up, this difference was not statistically significant in the ITT population ( $p=0.2613$ ); however, analysis of the eligible population as well as a multivariate analysis showed a significant survival benefit. Importantly, in post hoc analyses of these data, a prognostic model was created which was validated in several other studies. ${ }^{28-30}$ This treatment response model classifies patients into 4 risk categories based on the presence of $0,1,2$, or 3 of the 


\begin{tabular}{|c|c|c|c|c|c|c|c|c|c|}
\hline $\begin{array}{l}\text { Sampling } \\
\text { Frame }\end{array}$ & $\begin{array}{l}\text { Study Start } \\
\text { Date }\end{array}$ & $\begin{array}{l}\text { Study End } \\
\text { Date }\end{array}$ & Trial ID & $\begin{array}{l}\text { No. Patients } \\
\text { Vinflunine }\end{array}$ & $\begin{array}{l}\text { Median } \\
\text { Age }\end{array}$ & Male & Female & $\begin{array}{l}\text { No. Patients } \\
\text { Stage IV }\end{array}$ & $\begin{array}{l}\text { Median } \\
\text { Follow-up } \\
\text { (months) }\end{array}$ \\
\hline Retrospective & & & & 10 & & & & 10 & 10.5 \\
\hline Retrospective & I/I/20II & $|2 / 3| / 20 \mid 3$ & HGUCG & 36 & 68 & 31 & 5 & 36 & 16.8 \\
\hline Prospective & $5 / 15 / 2003$ & $8 / 15 / 2006$ & L00070 IN 302 & 253 & 64 & 292 & 78 & 370 & \\
\hline Prospective & $5 / 15 / 2003$ & $8 / 15 / 2006$ & L00070 IN 302 & 253 & 64 & & & & 42.9 \\
\hline Prospective & $5 / 15 / 2003$ & $8 / 15 / 2006$ & L00070 IN 302 & 253 & 64 & & & & \\
\hline Prospective & $4 / 15 / 2012$ & $1 / 15 / 2015$ & MAJA & 33 & 63 & & & & 7.2 \\
\hline Retrospective & $12 / 15 / 2009$ & $6 / 15 / 2013$ & & 102 & 67 & & & 102 & 8.9 \\
\hline Retrospective & $4 / 15 / 2010$ & $12 / 15 / 2012$ & & 45 & 68 & & & & \\
\hline Prospective & $11 / 15 / 2000$ & $9 / 15 / 2002$ & & 51 & 63 & 41 & 17 & 58 & \\
\hline Prospective & $2 / 15 / 2011$ & $8 / 15 / 2012$ & JASINTI & 69 & 70 & & & 69 & 25.9 \\
\hline Prospective & $2 / 15 / 2012$ & $7 / 15 / 2013$ & JASiMA & 19 & & & & & 9 \\
\hline Retrospective & $1 / 15 / 2010$ & $1 / 15 / 2015$ & & 10 & & & & 52 & \\
\hline Retrospective & $11 / 15 / 2012$ & $2 / 15 / 2013$ & CURVE & 134 & & & & & \\
\hline Retrospective & $2 / 15 / 2011$ & $6 / 15 / 2014$ & MOVIE & 84 & 72 & 71 & 13 & 84 & \\
\hline Retrospective & $2 / 15 / 2012$ & $3 / 15 / 2015$ & & 43 & 63.5 & 40 & 3 & 43 & 24 \\
\hline Prospective & $4 / 15 / 2012$ & $1 / 15 / 2015$ & MAJA & 45 & 64 & & & 88 & 12.2 \\
\hline Prospective & & & & 114 & 66 & 88 & 26 & & \\
\hline Prospective & $5 / 15 / 2011$ & $|2 /| 5 / 20||$ & NCTOI 265940 & 5 & 72 & 5 & 0 & 5 & \\
\hline Retrospective & $3 / 15 / 2010$ & $11 / 15 / 2013$ & & 37 & 67 & 36 & 1 & & 37 \\
\hline Prospective & $5 / 15 / 2003$ & $8 / 15 / 2006$ & L00070 IN 302 & 253 & 64 & 279 & 91 & 370 & 45.4 \\
\hline Prospective & & & & 77 & & & & & \\
\hline Retrospective & $2 / 15 / 2010$ & $3 / 15 / 2012$ & & 21 & 63 & 19 & 2 & 21 & \\
\hline Retrospective & $2 / 15 / 2010$ & $7 / 15 / 2013$ & & 100 & 68 & 72 & 28 & 100 & \\
\hline Ambispective & $4 / 15 / 2013$ & $4 / 15 / 2014$ & & 72 & 68 & 183 & 35 & & \\
\hline Retrospective & & & & 37 & 64 & 24 & 13 & 37 & \\
\hline Retrosective & $|/ I / 200|$ & $|2 / 3| / 20 \mid 3$ & & 40 & 67 & & & 40 & \\
\hline Retrosective & $1 / I / 2011$ & $|2 / 3| / 20||$ & & 134 & 65.3 & 119 & 15 & 134 & 17.6 \\
\hline Retrospective & $5 / 15 / 2010$ & $3 / 15 / 2014$ & & 19 & 66 & 18 & 1 & 19 & \\
\hline Prospective & $4 / 15 / 2011$ & $6 / 15 / 2014$ & & 16 & 62 & 13 & 3 & 16 & 5.2 \\
\hline Retrospective & $2 / 15 / 2011$ & $6 / 15 / 2014$ & MOVIE & 217 & 69 & 182 & 35 & 217 & \\
\hline Retrospective & $7 / 15 / 2005$ & $7 / 15 / 2014$ & & 71 & 66.8 & 65 & 6 & & 11.8 \\
\hline Prospective & $4 / 15 / 2012$ & $9 / 15 / 2013$ & MAJA & 20 & 65.6 & & & 46 & \\
\hline Prospective & $1 / 13 / 2015$ & $3 / 13 / 2017$ & IMvigor2II & 250 & 67 & 195 & 55 & 233 & 17.3 \\
\hline Prospective & $8 / 15 / 2010$ & $9 / 15 / 2011$ & NCTOII 03544 & 77 & 67 & 63 & 14 & 77 & 4.6 \\
\hline Prospective & & & & $15 \mid$ & 66 & 121 & 30 & $15 \mid$ & 11.9 \\
\hline
\end{tabular}

following risk factors: ECOG performance status ( 0 versus 1$)$, liver metastases (present versus absent), and hemoglobin $(<10$ versus $\geq 10 \mathrm{~g} / \mathrm{dl}$ ). In this study, median OS varied from 14.2 to 1.7 months depending on these risk factors, stressing the importance of patient selection in VIN as second-line therapy.

JASINT1 was the first study to examine first-line therapy doublets containing VIN in patients with good ECOG performance status $(0 / 1)$ and impaired renal function making them ineligible for cisplatin. Significantly less grade 3-4 hematologic AEs were reported with VG versus $\mathrm{VC}$ with similar DCR, ORR, and OS. ${ }^{11}$ To date, there have been no efficacy comparison studies of VIN combinations to current standard of first-line alternatives to cisplatin-containing regimens. We suggest a comparison study with carboplatin combinations as a logical next step in assessing this treatment as we are unable to assess comparative survival statistics at this time.
VIN maintenance therapy after first-line cisplatinbased treatment was shown in the JASiMA trial as well as by Aparicio et al to have an acceptable toxicity profile., ${ }^{9,31}$ Neutropenia, the most common AE, was reversible and noncumulative. The MAJA trial has shown favorable outcomes comparing VIN maintenance with BSC with 59\% disease progression and $43 \%$ patient death in the treatment arm versus $81 \%$ and $62 \%$, respectively, in the BSC control arm. ${ }^{10}$ Survival analysis in this trial is pending; however, this response benefit was demonstrated in the context of favorable tolerability.

Di Lorenzo et al evaluated third-line therapies including cyclophosphamide, platinum-containing regimens, paclitaxel, docetaxel, gemcitabine, and VIN in a total of 52 patients. ${ }^{23}$ In this setting, VIN showed inferior PFS and OS outcomes to cyclophosphamide as well as $18.75 \%$ higher absolute incidence of grade 3 and above AEs. Although a 


\begin{tabular}{|c|c|}
\hline Source & Proportion $(95 \% \mathrm{CI})$ \\
\hline Bellmunt et $\mathrm{al}^{8}$ & $0.09[0.06-0.13]$ \\
\hline Castellano et al ${ }^{35}$ & $0.25[0.17-0.34]$ \\
\hline Chirivella et al ${ }^{39}$ & $0.27[0.15-0.42]$ \\
\hline Culine et $\mathrm{al}^{5}$ & $0.18[0.08-0.31]$ \\
\hline De Santis et al ${ }^{11}$ & $0.48[0.36-0.60]$ \\
\hline Di Lorenzo et al 23 & $0.30[0.07-0.65]$ \\
\hline Facchini et $a^{27}$ & $0.12[0.04-0.25]$ \\
\hline Hegele et al ${ }^{44}$ & $0.19[0.05-0.42]$ \\
\hline Holmsten et al45 & $0.23[0.15-0.32]$ \\
\hline Hussain et al ${ }^{47}$ & $0.35[0.20-0.53]$ \\
\hline Medioni et al ${ }^{36}$ & $0.22[0.15-0.30]$ \\
\hline Moriceau et al48 & $0.32[0.13-0.57]$ \\
\hline Palacka et al ${ }^{26}$ & $0.12[0.02-0.38]$ \\
\hline Passalacqua et al ${ }^{49}$ & $0.13[0.09-0.18]$ \\
\hline Pistamaltzian et al50 & $0.13[0.06-0.23]$ \\
\hline Retz et al ${ }^{51}$ & $0.23[0.14-0.34]$ \\
\hline Vaughn et al ${ }^{6}$ & $0.15[0.09-0.21]$ \\
\hline Total (fixed effect) & $0.18[0.16-0.20]$ \\
\hline Total (random effects) & $0.21[0.16-0.26]$ \\
\hline Heterogeneity: $\chi_{16}^{2}=74$. & $72(P<0.01), R=79 \%$ \\
\hline
\end{tabular}

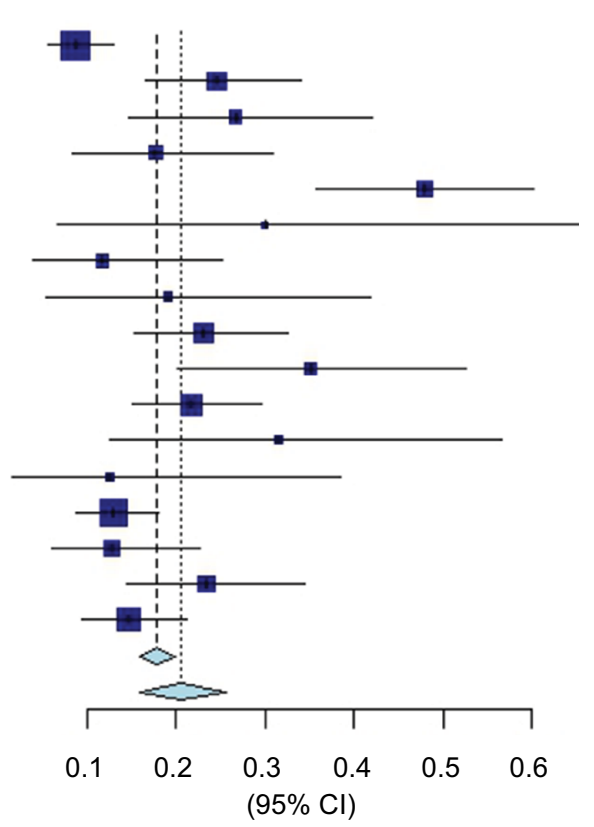

Figure 4 Forest plot of overall response rate data.

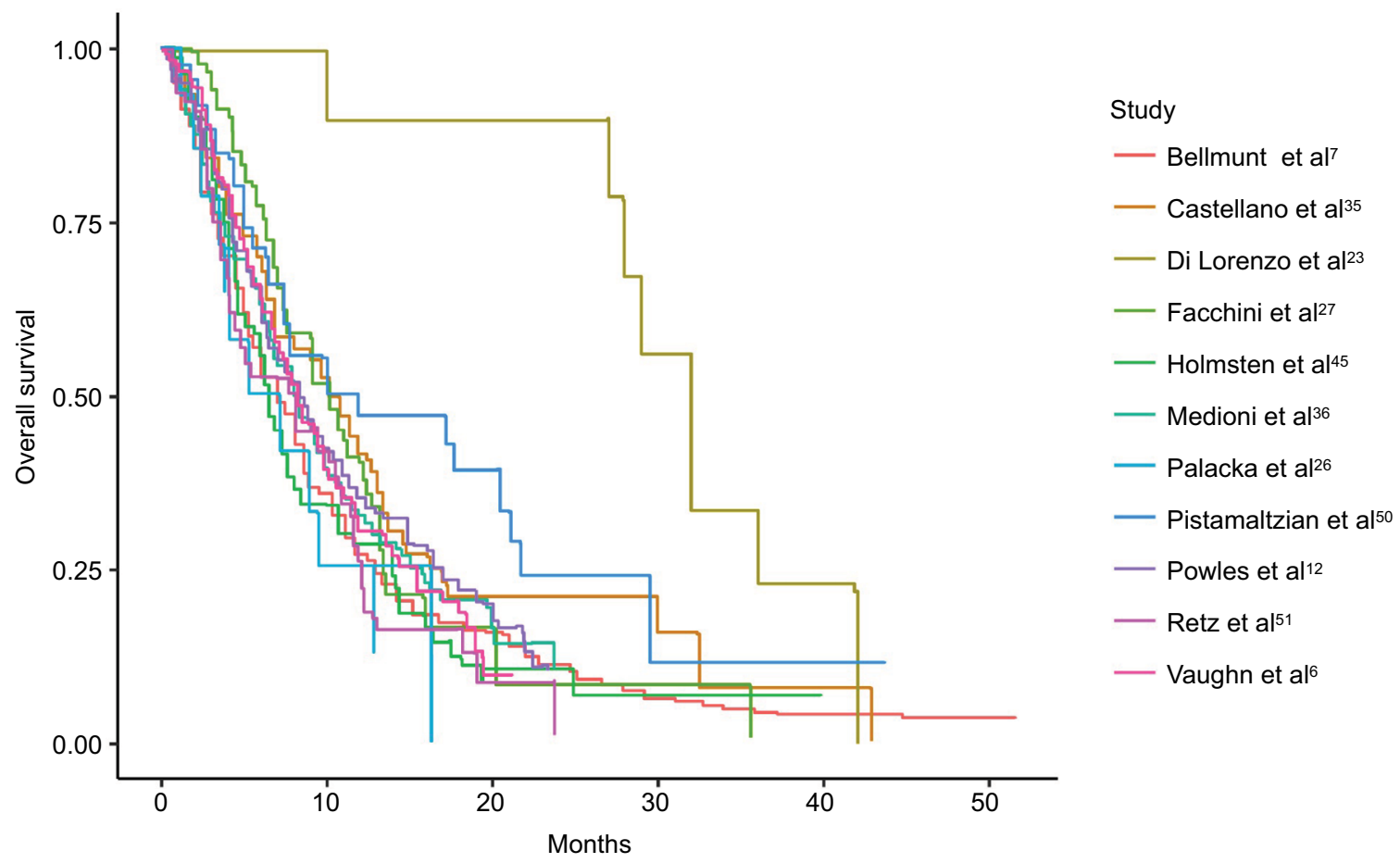

Figure 5 Pooled Kaplan-Meier estimate plots of overall survival.

better powered study would be beneficial, it does seem from this preliminary data that VIN does not seem active as a third-line choice.

It is exceedingly important to consider the cost to the health care system with the introduction of any new treatment.
Guglieri-Lopez et al performed an economic analysis of VIN, finding a median treatment cost of $€ 8,524$ per patient, $\mathrm{a} € 44,789$ per progression-free year gained, and $€ 22,750$ per life-year granted. ${ }^{32}$ We know that bladder cancer is the most expensive among cancer diagnoses per patient lifetime 


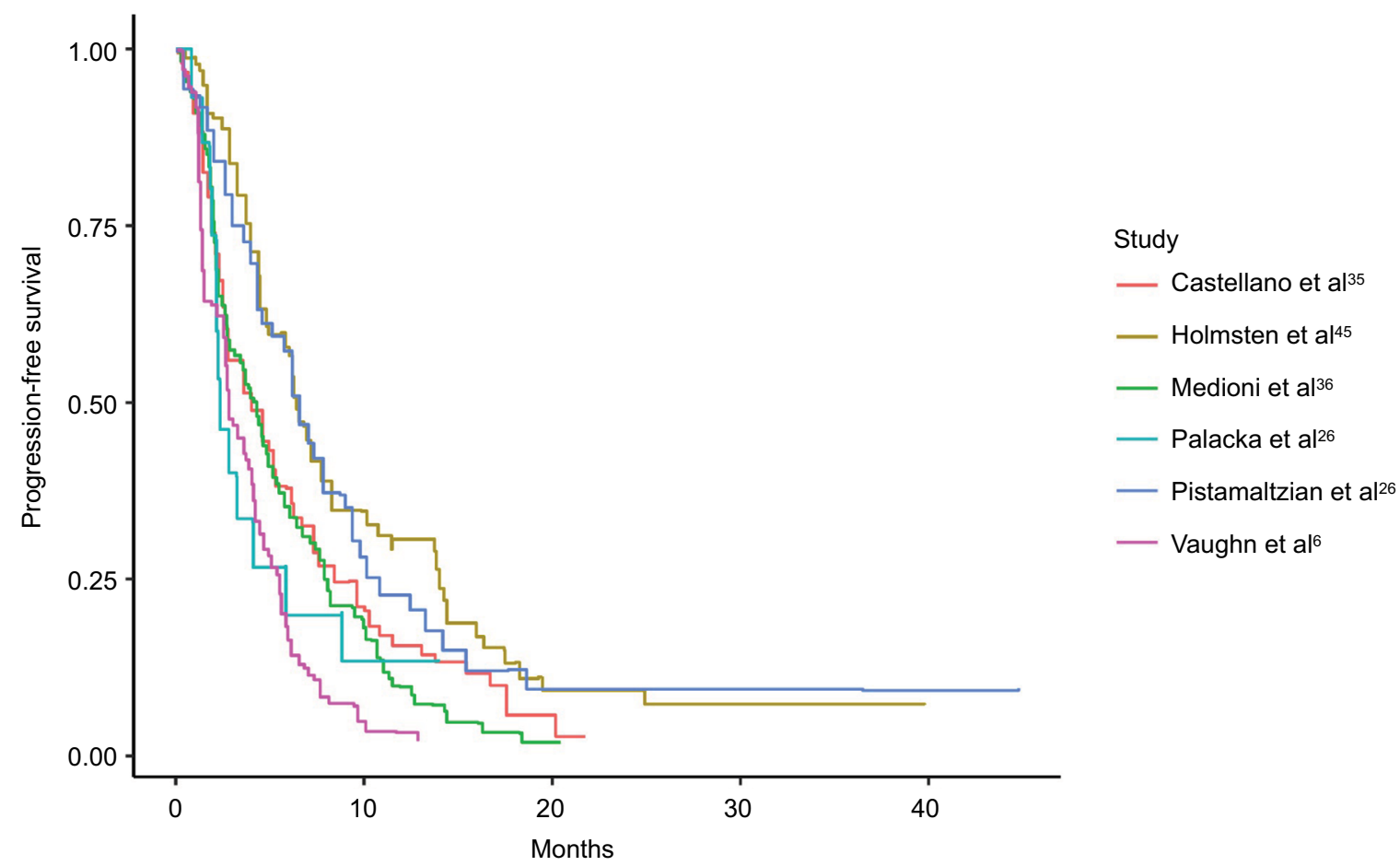

Figure 6 Pooled Kaplan-Meier estimate plots of progression-free survival.

Table 2 Non-hematologic adverse events of all CTCAE grades and grade 3-4

\begin{tabular}{|c|c|c|c|c|c|c|c|}
\hline $\begin{array}{l}\text { Non-hematologic adverse } \\
\text { events }\end{array}$ & $\begin{array}{l}\text { Number of } \\
\text { subjects }(\mathbf{N})\end{array}$ & $\begin{array}{l}\text { Number of } \\
\text { studies (k) }\end{array}$ & $I^{2}$ & $\begin{array}{l}\text { Fixed } \\
\text { effect }\end{array}$ & $95 \% \mathrm{Cl}$ & $\begin{array}{l}\text { Random } \\
\text { effect }\end{array}$ & $95 \% \mathrm{Cl}$ \\
\hline \multicolumn{8}{|l|}{ Adverse event (overall) } \\
\hline Fatigue & 1,000 & 10 & 93.9 & 0.426 & $0.395-0.456$ & $0.40 \mathrm{I}$ & $0.276-0.533$ \\
\hline Nausea & 715 & 7 & 82.1 & 0.357 & $0.323-0.393$ & 0.339 & $0.252-0.432$ \\
\hline Constipation & 1,112 & 12 & 96.7 & 0.31 & $0.283-0.337$ & $0.34 I$ & $0.207-0.489$ \\
\hline Alopecia & 416 & 4 & 71.5 & 0.273 & $0.232-0.317$ & 0.26 & $0.163-0.371$ \\
\hline Infusion-site reaction & 508 & 5 & 95.6 & 0.257 & $0.220-0.296$ & 0.34 & $0.023-0.789$ \\
\hline Vomiting & 854 & 9 & 90.4 & 0.249 & $0.220-0.278$ & 0.219 & $0.128-0.326$ \\
\hline Mucositis & 644 & 6 & 87.3 & 0.212 & $0.18 \mathrm{I}-0.244$ & 0.184 & $0.099-0.289$ \\
\hline Abdominal pain & 917 & 9 & 84.1 & 0.178 & $0.154-0.203$ & 0.168 & $0.101-0.247$ \\
\hline Myalgias & 567 & 5 & 8.8 & 0.154 & $0.125-0.185$ & 0.154 & $0.125-0.185$ \\
\hline Neuropathy & 805 & 7 & 92.5 & 0.151 & $0.127-0.177$ & 0.157 & $0.07 \mid-0.27 I$ \\
\hline \multicolumn{8}{|l|}{ Adverse event (grade 3-4) } \\
\hline Fatigue & $\mathrm{I}, 38 \mathrm{I}$ & 14 & 91.3 & 0.113 & $0.097-0.131$ & 0.102 & $0.053-0.164$ \\
\hline Constipation & $|, 63|$ & 19 & 76.9 & 0.086 & $0.073-0.100$ & 0.082 & $0.056-0.113$ \\
\hline Abdominal pain & 1,025 & 10 & 56.9 & 0.047 & $0.035-0.061$ & 0.046 & $0.028-0.070$ \\
\hline Myalgias & 667 & 6 & 4.7 & 0.025 & $0.015-0.038$ & 0.025 & $0.015-0.038$ \\
\hline Nausea & 870 & 10 & 42.4 & 0.023 & $0.014-0.034$ & 0.023 & $0.011-0.038$ \\
\hline Vomiting & 972 & 11 & 40.1 & 0.023 & $0.014-0.033$ & 0.022 & $0.012-0.036$ \\
\hline Mucositis & 773 & 9 & 56.2 & 0.02 & $0.011-0.031$ & 0.02 & $0.006-0.041$ \\
\hline Neuropathy & 974 & 10 & 81.7 & 0.015 & $0.008-0.024$ & 0.018 & $0.002-0.048$ \\
\hline Infusion-site reaction & 598 & 5 & 64 & 0.007 & $0.002-0.016$ & 0.007 & $0.000-0.025$ \\
\hline
\end{tabular}

Abbreviation: CTCAE, Common Terminology Criteria for Adverse Events.

and carries annual costs of $\$ 3.98$ billion in the US..$^{33,34}$ Although we are unable to directly compare this study to the cost-effectiveness data of other UC therapies, the high cost associated with VIN makes patient selection an extremely important factor, specifically when considering the modest survival benefit to patients in higher-risk categories.

As far as toxicity is concerned, VIN was associated with considerable rates of high-grade anemia and neutropenia. 
Table 3 Hematologic adverse events of all CTCAE grades and grade 3-4

\begin{tabular}{|c|c|c|c|c|c|c|c|}
\hline $\begin{array}{l}\text { Hematologic adverse } \\
\text { events }\end{array}$ & $\begin{array}{l}\text { Number of } \\
\text { subjects (N) }\end{array}$ & $\begin{array}{l}\text { Number of } \\
\text { studies (k) }\end{array}$ & $I^{2}$ & Fixed effect & $95 \% \mathrm{Cl}$ & Random effect & $95 \% \mathrm{Cl}$ \\
\hline \multicolumn{8}{|l|}{ Adverse event (overall) } \\
\hline Anemia & 1,058 & 12 & 98.4 & 0.653 & $0.624-0.681$ & 0.546 & $0.299-0.782$ \\
\hline Neutropenia & 1,058 & 12 & 97.6 & 0.513 & $0.483-0.543$ & 0.46 & $0.273-0.654$ \\
\hline Thrombocytopenia & 1,037 & 11 & 96.8 & 0.298 & $0.27 I-0.326$ & 0.255 & $0.125-0.412$ \\
\hline Febrile neutropenia & 815 & 8 & 70.4 & 0.06 & $0.045-0.078$ & 0.066 & $0.033-0.110$ \\
\hline \multicolumn{8}{|c|}{ Adverse event (grade 3-4) } \\
\hline Neutropenia & $|, 52|$ & 18 & 94.3 & 0.264 & $0.242-0.286$ & 0.246 & $0.165-0.338$ \\
\hline Anemia & 1,222 & 15 & 86.5 & 0.115 & $0.098-0.133$ & 0.102 & $0.059-0.154$ \\
\hline Febrile neutropenia & 995 & 12 & 38.6 & 0.055 & $0.042-0.070$ & 0.054 & $0.035-0.076$ \\
\hline Thrombocytopenia & 1,073 & 11 & 69.6 & 0.032 & $0.022-0.043$ & 0.03 & $0.013-0.054$ \\
\hline
\end{tabular}

Abbreviation: CTCAE, Common Terminology Criteria for Adverse Events.

While these conditions can come at the cost of the need for dose reduction and even treatment termination, it is important to consider that these AEs are often preventable with appropriate prophylaxis, or are treatable and reversible. ${ }^{35,36}$ Data suggest that overall AEs may be correlated with increased efficacy of treatment. ${ }^{37}$

\section{Conclusion}

VIN has been explored as a combination first-line treatment as well as a single-agent second-line, third-line, and maintenance therapy in advanced and metastatic UC. In first-line treatment of UC, either as a maintenance agent after cisplatin or as a primary combination therapy, VIN may be a promising alternative to current treatments. Further studies are needed to compare first-line combination VIN regimens to the current standard of care in order to assess long-term survival outcomes. Second- and third-line VIN monotherapy does provide a proven, although limited, survival benefit in platinum-refractory patients.

\section{Disclosure}

The authors report no conflicts of interest in this work.

\section{References}

1. Seront E, Machiels JP. Molecular biology and targeted therapies for urothelial carcinoma. Cancer Treat Rev. 2015;41(4):341-353.

2. De Santis M, Bellmunt J, Mead G, et al. Randomized phase II/III trial assessing gemcitabine/carboplatin and methotrexate/carboplatin/vinblastine in patients with advanced urothelial cancer who are unfit for cisplatin-based chemotherapy: EORTC Study 30986. J Clin Oncol. 2012;30(2):191-199.

3. Kruczynski A, Hill BT. Vinflunine, the latest vinca alkaloid in clinical development: a review of its preclinical anticancer properties. Crit Rev Oncol/Hematol. 2001;40(2):159-173.

4. Braguer D, Barret JM, McDaid H, Kruczynski A. Antitumor activity of vinflunine: effector pathways and potential for synergies. Semin Oncol. 2008;35(Suppl 3):S13-S21.

5. Culine S, Theodore C, De Santis M, et al. A phase II study of vinflunine in bladder cancer patients progressing after first-line platinumcontaining regimen. Br J Cancer. 2006;94(10):1395-1401.
6. Vaughn DJ, Srinivas S, Stadler WM, et al. Vinflunine in platinumpretreated patients with locally advanced or metastatic urothelial carcinoma: results of a large phase 2 study. Cancer. 2009;115(18):4110-4117.

7. Bellmunt J, Fougeray R, Rosenberg JE, et al. Long-term survival results of a randomized phase III trial of vinflunine plus best supportive care versus best supportive care alone in advanced urothelial carcinoma patients after failure of platinum-based chemotherapy. Ann Oncol. 2013;24(6):1466-1472.

8. Bellmunt J, Theodore C, Demkov T, et al. Phase III trial of vinflunine plus best supportive care compared with best supportive care alone after a platinum-containing regimen in patients with advanced transitional cell carcinoma of the urothelial tract. J Clin Oncol. 2009;27(27):4454-4461.

9. De Wit M, De Geeter P, Galli L, et al. Vinflunine maintenance treatment following first-line therapy of advanced urothelial carcinoma-results from the JASiMA trial. Eur J Cancer. 2015;51:S527.

10. Font A, Perez-Valderrama B, Virizuela JA, et al. Randomized, placebocontrolled phase II trial (MAJA): efficacy results of maintenance vinflunine after cisplatin chemotherapy $(\mathrm{CT})$ in patients with advanced urothelial carcinoma (UC)-SOGUG 2011-02. J Clin Oncol. 2016;34 (Suppl 15):4529.

11. De Santis M, Wiechno PJ, Bellmunt J, et al. Vinflunine-gemcitabine versus vinflunine-carboplatin as first-line chemotherapy in cisplatin-unfit patients with advanced urothelial carcinoma: results of an international randomized phase II trial (JASINT1). Ann Oncol. 2016;27(3):449-454.

12. Powles T, Loriot Y, Duran I, et al. IMvigor211: a phase III randomized study examining atezolizumab vs. chemotherapy for platinum-treated advanced urothelial carcinoma. Presented at: EACR-AACR-SIC Special Conference 2017: The Challenges of Optimizing Immuno- and Targeted Therapies: From Cancer Biology to the Clinic; June 24, 2017; Florence.

13. Eisenhauer EA, Therasse P, Bogaerts J, et al. New response evaluation criteria in solid tumours: revised RECIST guideline (version 1.1). Eur J Cancer. 2009;45(2):228-247.

14. Basch E, Reeve BB, Mitchell SA, et al. Development of the National Cancer Institute's patient-reported outcomes version of the common terminology criteria for adverse events (PRO-CTCAE). J Natl Cancer Inst. 2014;106(9).

15. Schwarzer G. Meta: an R package for meta-analysis. $R$ News. 2007;7(3):40-45.

16. Trikalinos TA, Dahabreh IJ, Lee J, Moorthy D. AHRQ methods for effective health care. Defining an Optimal Format for Presenting Research Needs. Rockville: Agency for Healthcare Research and Quality (US); 2011.

17. Viechtbauer W. Bias and efficiency of meta-analytic variance estimators in the random-effects model. J Educ Behav Stat. 2005;30(3):261-293.

18. Higgins JPT, Thompson SG. Quantifying heterogeneity in a metaanalysis. Stat Med. 2002;21(11):1539-1558.

19. Poisot $T$. The digitize package: extracting numerical data from scatterplots. $R J$. 2011;3(1):25-26.

20. Wickham H. ggplot2: Elegant Graphics for Data Analysis. New York: Springer-Verlag; 2009. 
21. Liberati A, Altman DG, Tetzlaff J, et al. The PRISMA statement for reporting systematic reviews and meta-analyses of studies that evaluate health care interventions: explanation and elaboration. J Clin Epidemiol. 2009;62(10):e1-e34.

22. Bellmunt J, Valderrama BP, Font A, et al. Maintenance vinflunine post cisplatin chemotherapy (CT) in patients with advanced urothelial carcinoma (UC): preliminary analysis of a randomized placebo controlled phase II trial (MAJA trial)-SOGUG 2011-02. J Clin Oncol. 2015;33(Suppl 15):4529.

23. Di Lorenzo G, Buonerba C, Bellelli T, et al. Third-line chemotherapy for metastatic urothelial cancer: a retrospective observational study. Medicine. 2015;94(51):e2297.

24. Marongiu M, Atzori F, Santoni M, et al. Efficacy of second and third-line chemotherapy in patients with advanced transitional-cell carcinoma: results of a national multicentre pooled analysis. Eur Urol Suppl. 2013;12(6):126.

25. Polo SH, Del Alba AG, Perez-Valderrama B, et al. Vinflunine maintenance therapy versus best supportive care after platinum combination in advanced bladder cancer: a phase II, randomized, open label, study (MAJA study, SOGUG 2011-02)-interim analysis on safety. J Clin Oncol. 2014;32(Suppl 4):359.

26. Palacka P, Mego M, Obertova J, Chovanec M, Sycova-Mila Z, Mardiak $\mathrm{J}$. The first Slovak experience with second-line vinflunine in advanced urothelial carcinomas. Klin Onkol. 2014;27(6):429-433.

27. Facchini G, Della Pepa C, Cavaliere C, et al. From clinical trials to the front line: vinflunine for treatment of urothelial cell carcinoma at the National Cancer Institute of Naples. Front Pharmacol. 2016;7:110.

28. Bellmunt J, Choueiri TK, Fougeray R, et al. Prognostic factors in patients with advanced transitional cell carcinoma of the urothelial tract experiencing treatment failure with platinum-containing regimens. $J$ Clin Oncol. 2010;28(11):1850-1855.

29. Di Palma M, Spaeth D, Guillot A, Médioni J, Théodore C. Prognostic factors of patients treaded by vinflunine for an advanced or metastatic urothelial carcinoma: results of the CURVE study. Eur J Cancer. 2013;49:S664.

30. Harshman LC, Fougeray R, Choueiri TK, et al. The impact of prior platinum therapy on survival in patients with metastatic urothelial cancer receiving vinflunine. Br J Cancer. 2013;109(10): 2548-2553.

31. Aparicio LA, Campelo R, Antolin S, et al. Safety and efficacy of vinflunine in long-time therapy of metastatic bladder cancer. Eur J Cancer. 2013;49:S672-S673.

32. Guglieri-Lopez B, Perez-Pitarch A, Porta-Oltra B, Ferriols-Lisart F, Climente-Marti M, Alos-Alminana M. Effectiveness, toxicity, and economic evaluation of vinflunine for the treatment of patients with transitional cell carcinoma in the Spanish outpatient setting. Anticancer Drugs. 2015;26(8):860-865.

33. Botteman MF, Pashos CL, Redaelli A, Laskin B, Hauser R. The health economics of bladder cancer: a comprehensive review of the published literature. Pharmacoeconomics. 2003;21(18):1315-1330.

34. Mariotto AB, Yabroff KR, Shao Y, Feuer EJ, Brown ML. Projections of the cost of cancer care in the United States: 2010-2020. J Natl Cancer Inst. 2011;103(2):117-128.

35. Castellano D, Puente J, de Velasco G, et al. Safety and effectiveness of vinflunine in patients with metastatic transitional cell carcinoma of the urothelial tract after failure of one platinum-based systemic therapy in clinical practice. BMC Cancer. 2014;14(1):779.

36. Médioni J, Di Palma M, Guillot A, Spaeth D, Théodore C. Efficacy and safety of vinflunine for advanced or metastatic urothelial carcinoma in routine practice based on the French multi-centre CURVE study. BMC Cancer. 2016;16:217.
37. Abola MV, Prasad V, Jena AB. Association between treatment toxicity and outcomes in oncology clinical trials. Ann Oncol. 2014;25(11):2284-2289.

38. Bamias A, Peroukidis S, Stamatopoulou S, et al. Utilization of Systemic Chemotherapy in Advanced Urothelial Cancer: A Retrospective Collaborative Study by the Hellenic Genitourinary Cancer Group (HGUCG). Clin Genitourin Cancer 2016;14(2):e153-e159.

39. Chirivella I, Grande E, Lopez-Criado P, et al. Vinflunine (VFL) as second-line chemotherapy for patients with transitional cell carcinoma of the urothelium (TCCU): A multicenter retrospective study. European Journal of Cancer 2013;49:S671.

40. Donini M, Passalacqua R, Montironi R, et al. Effectiveness and possible molecular factors predictive of clinical outcomes in patients with transitional cell carcinoma of the urothelial tract (TCCU) treated with VInfluninE: A multicenter retrospective study (MOVIE) of the gruppo oncologico Italiano Di Ricerca clinica (GOIRC). Annals of Oncology 2015;26:vi53-vi54.

41. George C, Vaughn DJ, Petrylak D, et al. Phase II study of single-agent vinflunine in platinum-refractory transitional cell carcinoma of the urotheliurn (TCCU). Ejc Supplements 2007;5(4):309-309.

42. Gerullis H, Eimer C, Ecke TH, Georgas E, Arndt C, Otto T. Combined treatment with pazopanib and vinflunine in patients with advanced urothelial carcinoma refractory after first-line therapy. Anticancer Drugs 2013;24(4):422-425.

43. Hegele A, De Geeter P, Goebell PJ, et al. Response to vinflunine treatment after prior platinum-based chemotherapy in patients with advanced urothelial carcinoma independent of primary tumor location. Journal of Clinical Oncology 2015;33(15).

44. Hegele A, Goebell P, Matz U, Neuhaus T. Monotherapy with intravenous vinflunine in patients with advanced or metastatic urothelial cancer after failure of a platinum-containing regimen: a retrospective analysis of German routine data. Urol Int 2014;92(2):174-179.

45. Holmsten K, Dohn L, Jensen NV, et al. Vinflunine treatment in patients with metastatic urothelial cancer: A Nordic retrospective multicenter analysis. Oncol Lett 2016;12(2):1293-1300.

46. Houede N, Locker G, Lucas C, et al. Epicure: a European epidemiological study of patients with an advanced or metastatic Urothelial Carcinoma (UC) having progressed to a platinum-based chemotherapy. BMC Cancer 2016;16(1):752.

47. Hussain S, Jackson R, Chauhan S, et al. VICTOR: Vinflunine in advanced metastatic transitional cell carcinoma of the urothelium (TCCU): A retrospective analysis of the use of Vinflunine in a multicentre real life setting as second line chemotherapy through the free of charge programme (FOCP) for patients in the UK. European Journal of Cancer 2015;51:S523.

48. Moriceau G, Vallard A, Rivoirard R, et al. Real-World Vinflunine Outcomes in Bladder Cancer in a Single-Institution Study: Moving Beyond Clinical Trials. Clin Genitourin Cancer 2015;13(6):588-592.

49.Passalacqua R, Lazzarelli S, Montironi R, et al. Vinflunine (VFL) in patients (pts) with metastatic transitional cell carcinoma of the urothelial tract (mTCCU): Clinical outcome and prognostic factors in a nationwide, real-life setting (MOVIE trial). Journal of Clinical Oncology 2016;34.

50. Pistamaltzian N, Tzannis K, Pissanidou V, et al. Treatment of relapsed urothelial bladder cancer with vinflunine: real-world evidence by the Hellenic Genitourinary Cancer Group. Anticancer Drugs 2016;27(1):48-53.

51. Retz M, de Geeter P, Goebell PJ, Matz U, de Schultz W, Hegele A. Vinflunine in routine clinical practice for the treatment of advanced or metastatic urothelial cell carcinoma - data from a prospective, multicenter experience. BMC Cancer 2015;15:455. 


\section{Publish your work in this journal}

Core Evidence is an international, peer-reviewed open-access journal evaluating the evidence underlying the potential place in therapy of drugs throughout their development lifecycle from preclinical to post launch. The focus of each review is to evaluate the case for a new drug or class in outcome terms in specific indications and patient groups.

Submit your manuscript here: https://www.dovepress.com/core-evidence-journal
The manuscript management system is completely online and includes a very quick and fair peer-review system, which is all easy to use. Visit http://www.dovepress.com/testimonials.php to read real quotes from published authors. 